REDES- Revista hispana para el análisis de redes sociales

Vol.20,\#6, Junio 2011

http://revista-redes.rediris.es

\title{
Una definición estructural de capital social
}

José I. García-Valdecasas Medina

Department of Sociology. University of Leicester

\section{Resumen}

Este trabajo es una revisión de una definición de capital social que conecta la estructura topológica de las redes sociales -donde los individuos están insertos- con los recursos disponibles para dichos individuos en tales redes. El capital social, tal como aquí se defiende, consiste en una serie de recursos que los individuos pueden obtener a partir de las estructuras de las redes sociales. Dos de los recursos más importantes son la información que fluye por las redes y las obligaciones de reciprocidad que pueden generarse de la confianza mutua entre los agentes de la misma red. Ambos aspectos del capital social -información y reciprocidad- están íntimamente relacionados con la estructura de las redes: por un lado, el flujo de información depende de ciertas configuraciones estructurales (brokerage) caracterizadas por bajas longitudes de paso; y, por otro lado, las obligaciones de reciprocidad dependen de otras distribuciones estructurales (closure) determinadas por altos coeficientes de clustering.

Palabras claves: Confianza, Información, Brokerage, Closure, Estructura

\begin{abstract}
This paper is a review of a definition of social capital that relates the topological structure of social networks, in which individuals are embedded, to the resources available to individuals in such networks. Social capital, as defined here, consists of a series of resources that individuals can obtain from the structure of social networks. Two of the most important resources are information flowing through networks and obligations of reciprocity which can come out of the mutual confidence between agents in the same network. Both aspects of social capital information and reciprocity- are closely related to the structure of networks: firstly, the flow of information depends on certain structural configurations (brokerage) characterized by low path length; and, secondly, the obligations of reciprocity depend on other structural distributions (closure) characterized by high clustering coefficient.
\end{abstract}

Keywords: Trust, Information, Brokerage, Closure, Structure 


\section{Introducción}

La idea central defendida en este trabajo de investigación es sumamente simple: la estructura de las redes sociales importa. La estructura de las redes sociales puede generar capital social y el capital social puede producir tanto beneficios públicos como privados. Me centraré básicamente en dos aspectos del capital social, a saber: el potencial informativo que fluye por las redes sociales (Burt, 1992; 2005; Granovetter, 1973) y las obligaciones de reciprocidad derivadas de las relaciones de confianza entretejidas en las redes sociales (Ostrom y Walker, 2005; Herreros, 2002; Gambetta, 2000; Coleman, 1990; Bourdieu, 1986). Conviene aclarar desde el primer momento dos cuestiones importantes: el capital social no son redes sociales, pero sin redes sociales no hay capital social; y, en segundo lugar, el capital social no siempre produce beneficios, sino que también podría crear perjuicios (Levi, 1996).

Son muchas las investigaciones que pretenden poner de manifiesto las ventajas privadas que pueden generar las redes sociales (Granovetter, 1973; 1995; Erickson, 2001; Putnam, 2003; Kawashi, Subramanian y Kim, 2007; Layard, 2005). Algunos estudios indican, por ejemplo, que la probabilidad de que alguien encuentre trabajo depende más del tamaño de las redes sociales de vínculos débiles en las que se está inserto que de los títulos académicos que se posea (Granovetter, 1973; 1995). El dicho "no es sólo lo que conoces sino a quién conoces" sugiere que las redes sociales a las que pertenecen los individuos pueden ser tan o más importantes que la formación académica que se tenga a la hora de buscar un empleo. Algunos investigadores llegan incluso más lejos al defender que los ingresos están más determinados por la extensión de las relaciones sociales que por el nivel educativo (Erickson, 2001). Otros trabajos, por citar sólo algunos ejemplos más, analizan el poderoso efecto de las redes sociales sobre el logro educativo (Putnam, 2003) o sobre la salud física y psíquica de las personas (Kawashi, Subramanian y Kim, 2007). La literatura también señala que el capital social que se puede generar en las redes sociales es más importante para el bienestar subjetivo de las personas (la felicidad) que los bienes materiales (Layard, 2005). De aquí que la pertenencia a redes sociales permita pronosticar la probabilidad de la felicidad humana mejor que la posesión de capital económico; y es que el mejor predictor de la felicidad de una persona es la amplitud y la profundidad de sus contactos sociales. 
Otros estudios, en cambio, intentan poner de relieve las ventajas públicas que pueden promover las redes sociales (Woolcock, 1998; Putnam, 1993; 1995; 2000; Putnam y Goss, 1993; Narayan, 2002). El capital social que dichas redes pueden generar desempeña un papel crucial en muchas facetas de la vida social, política y económica de las sociedades (para una discusión más amplia, véase Andréu, 2005). Por ejemplo, puede reducir los costes de transacción propios de todo intercambio de mercancías y, por consiguiente, estimular el desarrollo económico (Woolcock, 1998); permite un mayor control sobre los gobernantes favoreciendo el funcionamiento de la democracia (Putnam, 1993; 1995; 2000; Putnam y Goss, 1993); puede ser un factor clave en la reducción de la delincuencia; o puede ser un elemento importante para la erradicación de la pobreza (Narayan, 2002), entre otros muchos aspectos.

Así pues, sociedades que poseen redes sociales con elevados niveles de capital social tienen menos problemas que otras sociedades deficitarias en redes sociales y con menos recursos de capital social, como intentan acreditar numerosas investigaciones (Putnam, 1993; 1995; 2000). Por poner un paralelismo biológico, de igual manera que el potente sistema inmunitario de los mamíferos reduce las posibilidades de desarrollar cáncer y protege a los individuos contra muchas enfermedades infecciosas, sociedades con elevadas reservas de capital social y con redes sociales valiosamente trabadas son menos propicias a sufrir problemas sociales. Las redes sociales y el capital social que se puede generar desde dichas redes nos protegen contra diversas patologías sociales tales como la pobreza, la delincuencia, el oportunismo político, el descontento o las dolencias físicas y mentales que pueden sufrir los individuos, entre otros muchos aspectos. Asimismo podemos afirmar que, de la misma forma que el descenso de la actividad del sistema inmunológico en los seres humanos no es la causa directa del cáncer pero lo posibilita, el declive de las reservas capital social en la sociedad tampoco es el origen último de los problemas sociales, pero sin duda los permite.

Si esto es así, la pregunta acerca de cómo aumentar los niveles de capital social puede resultar crucial tanto para el progreso económico como para el funcionamiento democrático, así como para el desarrollo integral de los individuos. Ahora bien, responder a la cuestión de cómo mejorar los niveles de capital social que tan buenos beneficios parece poseer supone responder previamente a otra pregunta: qué es el capital social. Este artículo está dedicado a responder a esta cuestión clave. 


\section{Definición de capital y tipos de capital: físico, humano y social}

Entender qué es el capital social implica clarificar en primer lugar el concepto de capital. El término "capital" hace referencia a los recursos que se invierten para obtener beneficios. Según Karl Marx ([1849] 1933) el capital emerge en las relaciones sociales de explotación entre capitalistas y trabajadores. En el esquema marxiano, la clase explotadora recoge el valor añadido generado por el trabajo proporcionado por la clase explotada; así pues, los burgueses -que poseen los medios de producción- pueden acumular capital, mientras que los proletarios no. Sin embargo, para Adam Smith (1937) el capital incluye todas las habilidades prácticas y adquiridas por parte de los individuos; desde esta perspectiva, el capital no sólo recae en los capitalistas sino también entre los trabajadores. Por tanto, los trabajadores se han convertido también en capitalistas no porque posean medios de producción, sino por tener habilidades y conocimientos de valor económico.

Se podría, pues, distinguir entre un capital físico -que puede rastrearse hasta los análisis de Marx- y un capital humano, cuyos orígenes se remontan a la obra de Smith. A diferencia del capital físico, el capital humano es el valor añadido a los trabajadores cuando dichos individuos adquieren habilidades, conocimientos y otros recursos útiles para el proceso de producción. Dicho capital humano se puede medir típicamente por el nivel educativo, formación específica y experiencia de trabajo.

La teoría del capital de Marx y la de Smith tienen en común la definición de capital como aquellos recursos que se invierten para obtener beneficios; sin embargo, se distancian tanto en sus consideraciones acerca de quién puede o no poseer dicho capital como en sus visiones de la estructura social. Según Marx, la estructura social es un rígido sistema de dos clases sociales: los capitalistas que pueden acumular capital y los trabajadores que no. Desde el punto de vista de Smith, sin embargo, la estructura social se contempla como una jerarquía fluida de muchos grados de capitalistas con diferentes oportunidades y motivaciones para la adquisición de capital humano.

Es importante notar que hay un cambio de perspectiva desde el nivel macro usado por Marx hasta el nivel micro empleado por Smith (Lin, 2001:17). Mientras que Marx concibe el capital cómo parte del proceso de explotación en la sociedad, Smith se centra en el capital adquirido por los individuos a fin de obtener beneficios. Sin embargo, se debe tener en cuenta tanto la acción de los individuos que genera cambios sociales (la "mano invisible" de Smith) como las estructuras de interacción que influyen en las acciones de los individuos (las estructuras de explotación de Marx) si se quiere explicar fenómenos sociales. 
El capital humano puede ser concebido, pues, como un conjunto de recursos que se invierten en los actores individuales con el deseo de obtener beneficios. Sin embargo, el capital social puede ser definido como una serie de recursos que se invierten en relaciones sociales con la esperanza de adquirir ciertas ventajas. Se podría distinguir, pues, entre recursos personales y recursos sociales (Lin, 1982): los recursos personales son propios de los actores individuales e incluyen tanto bienes simbólicos (tales como títulos, diplomas y otros certificados) como bienes materiales. Por el contrario, los recursos sociales están incrustados en las redes sociales, y sólo se puede acceder a ellos a través de las conexiones sociales. El coche prestado por un amigo o el trabajo conseguido a través de un antiguo compañero de clase del padre serían ejemplos de recursos sociales. Es importante subrayar que los recursos sociales, tanto por cantidad como por calidad, pesan más que los recursos personales en su utilidad potencial para los individuos (Lin, 2001).

Así pues, el capital social consiste en un conjunto de recursos insertos en las redes sociales en los que se puede invertir cuando los actores desean aumentar la probabilidad de éxito en sus acciones (Lin, 2001:24). Al igual que el capital humano, el capital social es una serie de recursos en los que se invierte para obtener beneficios; pero a diferencia del capital humano que representa una inversión en conocimientos y habilidades, el capital social implica una inversión en redes sociales.

Los recursos disponibles en las redes sociales pueden mejorar el resultado de la acción de los actores que pertenecen a tales redes de dos maneras: en primer lugar, proporcionando información muy útil sobre las necesidades del mercado y sobre diferentes oportunidades para tales actores; y en segundo lugar, reforzando la identidad, el reconocimiento y la confianza necesarias para mantener la salud física y psíquica de dichos actores.

En este trabajo de investigación se consideran dos elementos claves del capital social en tanto que recursos insertos en las redes sociales, a saber, el potencial informativo que fluye a lo largo de dichas redes y las obligaciones de reciprocidad (obtener favores a cambio de favores) que pueden surgir de la confianza mutua entre los actores que pertenecen a la misma red social (Herreros, 2002:20-21). Mientras que las redes sociales de alta densidad y cerradas (Coleman, 1990; Bourdieu, 1986) constituidas por vínculos fuertes son necesarias para poder generar confianza, los puentes entre los grupos sociales formados por vínculos débiles son fundamentales para el flujo de información (Burt, 1992; Granovetter, 1973). 


\section{Diferentes definiciones de capital social (Figura 1)}

Se puede concebir el capital social como una serie de recursos disponibles para los individuos derivados de su participación en redes sociales (Lin, 2001; Coleman, 1988; 1990; Bourdieu, 1986). Este artículo se centra en dos aspectos esenciales del capital social: la información que recorre la estructura de las redes sociales y las obligaciones de reciprocidad que pueden generarse de la confianza mutua entre los individuos pertenecientes a la misma estructura de red. Los sujetos no pueden acceder a dichos recursos de capital social si no participan de alguna manera en redes sociales. Así pues, un individuo aislado, que no participe en ninguna red social, no podrá disfrutar de los recursos del capital social.

Sin embargo, no todos los autores comparten esta definición de capital social. Ciertos autores (Paxton, 1999; Newton, 1997) no sostienen una definición estructural del capital social como la descrita anteriormente, sino una definición culturalista. Mientras que desde el punto de vista estructural el capital social puede ser un fenómeno objetivo y medible, desde la perspectiva culturalista es un fenómeno subjetivo y difícilmente medible constituido por "un conjunto de valores y actitudes que poseen los ciudadanos y que determinan cómo se comportan unos con otros" (Herreros, 2002:22). Esta serie de valores se refieren a virtudes cívicas tales como la fraternidad, la confianza generalizada o la cooperación, por ejemplo. Una sociedad cuyos ciudadanos posean dichas actitudes será una sociedad más sana y más abierta que otra que no las posea. Así pues, el capital social es, desde esta perspectiva culturalista, una serie de valores y actitudes que poseen los individuos y que tienen consecuencias beneficiosas tanto para los individuos particulares como para el resto de la sociedad (para una crítica de esta perspectiva, véase Herreros, 2002:22-35).

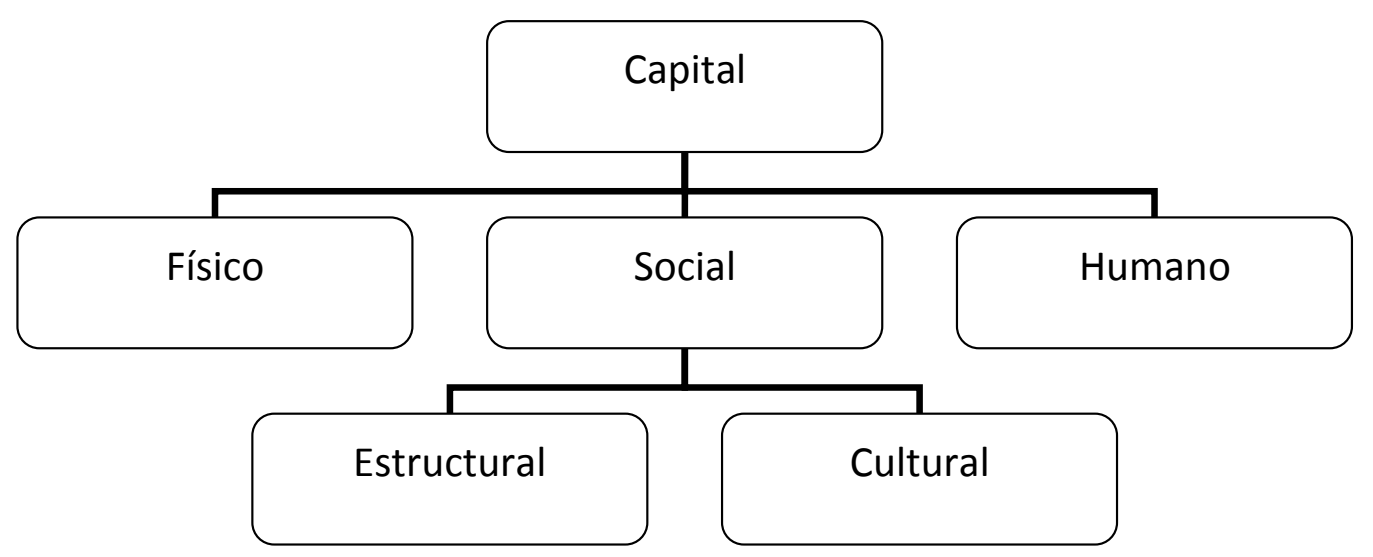

Figura 1. Diferentes tipos de capital y distintas concepciones de capital social. 
Antes de proponer una definición operativa de capital social, sería importante analizar algunas de las definiciones que han tenido gran repercusión en la discusión sobre la concepción de capital social (Portes, 1998). El concepto aparece por primera vez en un escrito de L. J. Hanifan en 1916 (Putnam, 2000:19). Este maestro rural y reformador social estadounidense llegó a la conclusión de que los graves problemas sociales, políticos y económicos que afligían a las comunidades donde trabajaba sólo podían resolverse reforzando las redes de solidaridad entre sus ciudadanos. Hanifan acuñó la expresión capital social para referirse a:

"esos elementos intangibles que cuentan sumamente en la vida diaria de las personas, a saber, la buena voluntad, la camaradería, la comprensión y el trato social entre individuos y familias" (citado en Putnam y Goss, 2003:10).

Así pues, al hablar de capital social este autor se refiere más a un conjunto de valores que a una serie de recursos, por lo que parece estar más cerca de una definición culturalista del capital social que de una concepción estructuralista. También subrayó las ventajas tanto públicas como privadas que genera el capital social:

"la comunidad en su conjunto se beneficiará de la cooperación de todas sus partes, mientras que el individuo encontrará al asociarse las ventajas de la ayuda, la compresión y la camaradería de sus vecinos" (citado en Putnam y Goss, 2003:10).

Otros autores como Michael Woolcock y Deepa Narayan (2000), por el contrario, defienden más bien una concepción estructuralista de capital social al afirmar que:

"la idea básica del capital social es que la familia, los amigos y los compañeros de una persona constituyen un recurso importante al que recurrir en una crisis, del que disfrutar por sí mismo y del que servirse para conseguir ventajas materiales. Además, esto, que es cierto para los individuos, vale también para los grupos. Las comunidades con recursos variados de redes sociales y asociaciones cívicas se encuentran en una posición más sólida para hacer frente a la pobreza y la vulnerabilidad, resolver disputas y sacar partido a oportunidades nuevas" (Woolcock y Narayan, 2000:226).

También estos autores ponen de manifiesto las ventajas tanto públicas como privadas que proporcionan el capital social. 
No obstante, el desarrollo del concepto "capital social" y su actual influencia en las ciencias sociales se debe fundamentalmente a los estudios de Pierre Bourdieu (1986), James Coleman (1988; 1990), Nan Lin (2001) y Robert Putnam, (1993; $1995 ;$ 2000). La definición estructural de capital social se deriva fundamentalmente de los trabajos de Bourdieu, Coleman y Lin. Dichos autores definen el capital social como un conjunto de recursos disponibles para los individuos derivados de su participación en redes sociales. Para Bourdieu (1986), el capital social es:

"la acumulación de recursos reales o potenciales que están unidos a la posesión de una red duradera de relaciones más o menos institucionalizadas de reconocimiento mutuo" (Bourdieu, 1986:248).

Es decir, el sociólogo francés concibe el capital social como una forma de capital poseído por los miembros de una red social, $y$, a través de de las conexiones sociales, dichos miembros de la red pueden hacer uso de tal capital social. Además, para Bourdieu -y a diferencia de Coleman- el capital social es un mero disfraz del capital económico:

"el capital económico es la raíz de todos los otros tipos de capital" y "todos los tipos de capital son reductibles en última instancia a capital económico" (Bourdieu, 1986:252).

Según Coleman (1990:302), el capital social es "un aspecto de la estructura social que facilita ciertas acciones de los individuos que están situados dentro de esa estructura". Si algún aspecto estructural sirve a los individuos para alcanzar la finalidad de sus acciones, entonces dicho aspecto es capital social, siendo los recursos derivados de las relaciones sociales un aspecto importante de la estructura social. Así pues, para el sociólogo estadounidense, el capital social es una serie de recursos reales o potenciales ganados a través de las relaciones sociales "que hacen posible el logro de ciertos fines que no serían alcanzados en su ausencia" (Coleman, 1990:302). Además, dicho capital social no es fungible a través de las acciones de los individuos. Así pues, tanto en la definición de capital social de Bourdieu como en la de Coleman existe una clara referencia a los términos recurso y estructura, por lo que ambas definiciones pueden ser calificadas de estructuralistas.

Por el contrario, en la definición de capital social de Putnam se pueden encontrar elementos que podrían ser adscritos tanto a la definición estructuralista como a la culturalista (Herreros, 2002:22). Para Putnam, por una parte, el capital social consiste en determinadas características que pueden poseer los ciudadanos como la posesión de virtudes cívicas, y en este sentido su definición está más cerca de la 
perspectiva culturalista (Putnam, 1993; 1995). Pero, por otra parte, también incluye a las redes sociales como un elemento crucial del capital social, y de esta manera se acerca más a la concepción estructuralista (Putnam, 2000). En su análisis sobre el declive del capital social en los EEUU considera el capital social como "las redes sociales y las normas de reciprocidad y confianza que se derivan de ellas" (citado en Herreros, 2002:22). Combinar ambas concepciones -la estructural y la cultural- en una misma definición impide un análisis más pormenorizado del término y dificulta sin duda responder a la pregunta crucial de cómo elevar los niveles de capital social.

Este artículo mantiene una visión estructural y objetiva del capital social entendido como un conjunto de recursos disponibles para los individuos tales como información y obligaciones de reciprocidad derivados de la participación de dichos individuos en las estructuras de las redes sociales, sin asumir -por el contrario- una visión cultural y subjetiva del capital social considerado como una colección de valores y actitudes tales como fraternidad, confianza generalizada, cooperación y otros valores similares.

\section{Definición estructural de capital social}

Podría argumentarse a favor de la importancia que tiene la estructura de las redes sociales a la hora de explicar las dinámicas que tienen lugar en el propio seno de dichas redes (González-Bailón, 2006). Numerosas investigaciones muestran el papel crucial que desempeña la estructura de las redes en multitud de procesos sociales, por ejemplo en la difusión de la innovación tecnológica, la expansión de los nuevos movimientos sociales, la propagación de las preferencias políticas o la búsqueda de empleo (Watts, 2003; Barabási, 2002; Granovetter, 1973; Coleman, Katz y Menzel, 1957). En este apartado, en particular, se define muy brevemente el concepto red social y se muestra cómo las estructuras de redes sociales son capaces de generar capital social.

La definición convencional de red social entendida como un conjunto de actores sociales unidos entre sí a través de relaciones sociales es un punto de partida bastante útil. Los actores sociales pueden ser de muy diversos tipos: individuos, grupos, empresas, clases, estados, etc., y las relaciones sociales también pueden ser de muy distintas clases: relaciones de amistad, relaciones de poder, transferencias de recursos, influencia política, etc. (Molina, 2001; Requena, 1989). 
Por otro lado, desde un punto de vista matemático, un grafo consiste en un conjunto de puntos o nodos y un conjunto de líneas o vínculos que conectan pares de nodos. Conviene no confundir grafos con redes sociales: los grafos (el modelo) no son las redes sociales (la realidad), pero los grafos sí pueden representar a las redes sociales. Es decir, los puntos pueden representar a los actores sociales y las líneas a las relaciones sociales, y, asimismo, las propiedades matemáticas de los grafos representan características estructurales de las redes sociales (Wasserman y Faust, 1994; Hanneman y Riddle, 2005). En realidad, un grafo puede ser considerado como la representación estática de una red social -una entidad siempre en continuo cambio-.

El capital social puede emerger no sólo en redes constituidas por segmentos donde los nodos están fuertemente interconectados entre sí (Coleman, 1990; Bourdieu, 1986), es decir, en estructuras caracterizadas por altos coeficientes de clustering (C), sino también en redes donde ciertos nodos facilitan las conexiones entre segmentos aislados entre sí (Burt, 2005; Granovetter, 1973), esto es, en estructuras definidas por bajas longitudes de paso (L). Así pues, el capital social puede ser generado por dos tipos de estructuras diferentes (figura 2): redes densas y cerradas ( $C$ altos), donde se puede forjar la identidad personal, generar confianza para la toma de decisiones y facilitar el intercambio de favores entre individuos; y redes fluidas y abiertas ( $L$ bajos), donde puede fluir la información crucial para la realización de los fines de los individuos y generarse nuevas ideas $y$ comportamientos. Al primer aspecto estructural se le denomina closure (cierre) y al segundo brokerage (intermediación) (Burt, 2005). Este artículo se centra precisamente en estos dos aspectos del capital social: reciprocidad y confianza generada mediante el closure, e información y nuevas ideas difundidas a través del brokerage (ver también el trabajo de Velásquez y Rey, 2007).

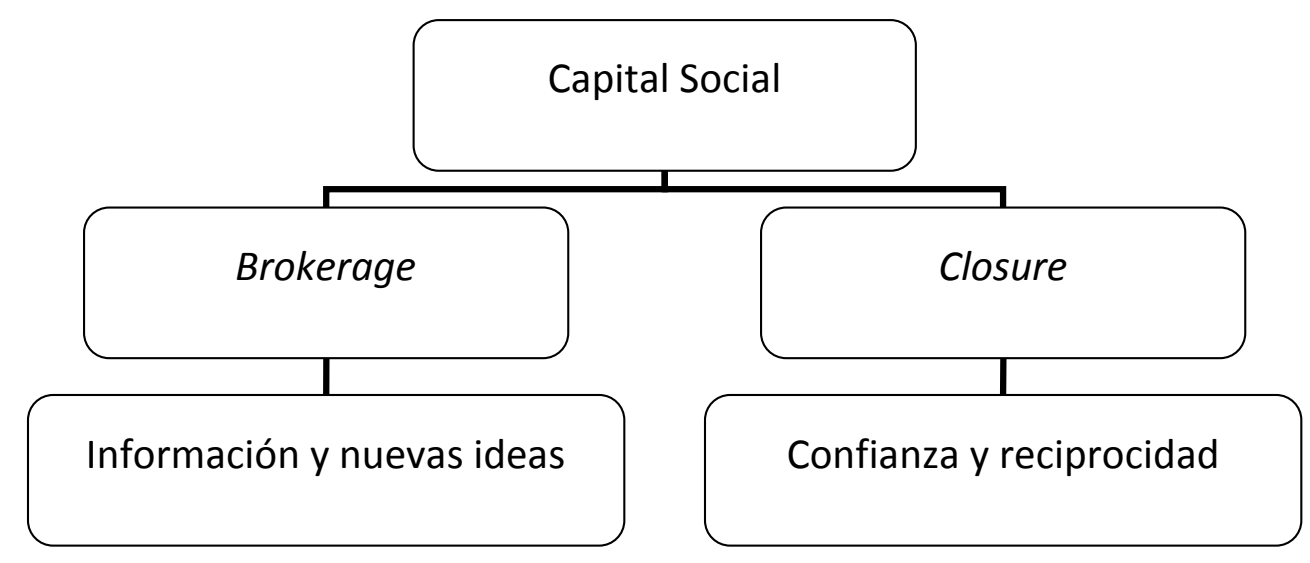

Figura 2: Diferentes aspectos del capital social. 


\section{a) Brokerage}

En la figura 3 se puede observar un grafo que representa a una red social en un momento determinado del tiempo. Las líneas continuas simbolizan a los vínculos fuertes y las líneas discontinuas a los débiles ${ }^{1}$.

Muchas redes sociales están caracterizadas por un conjunto de clusters (grupos de nodos internamente muy conectados) y por algunos vínculos ocasionales entre los clusters relativamente aislados (cuando un individuo tiene un amigo, conocido o colega en otro cluster). Es decir, las redes sociales son mundos pequeños (Watts, 2003) con coeficientes de clustering altos (debidos a los clusters) y longitudes de paso bajas (a causa de los puentes entre clusters). En este grafo se pueden observar 3 clusters: A, B y C, que son segmentos densos en el sentido de que las relaciones son más densas dentro que fuera del grupo.

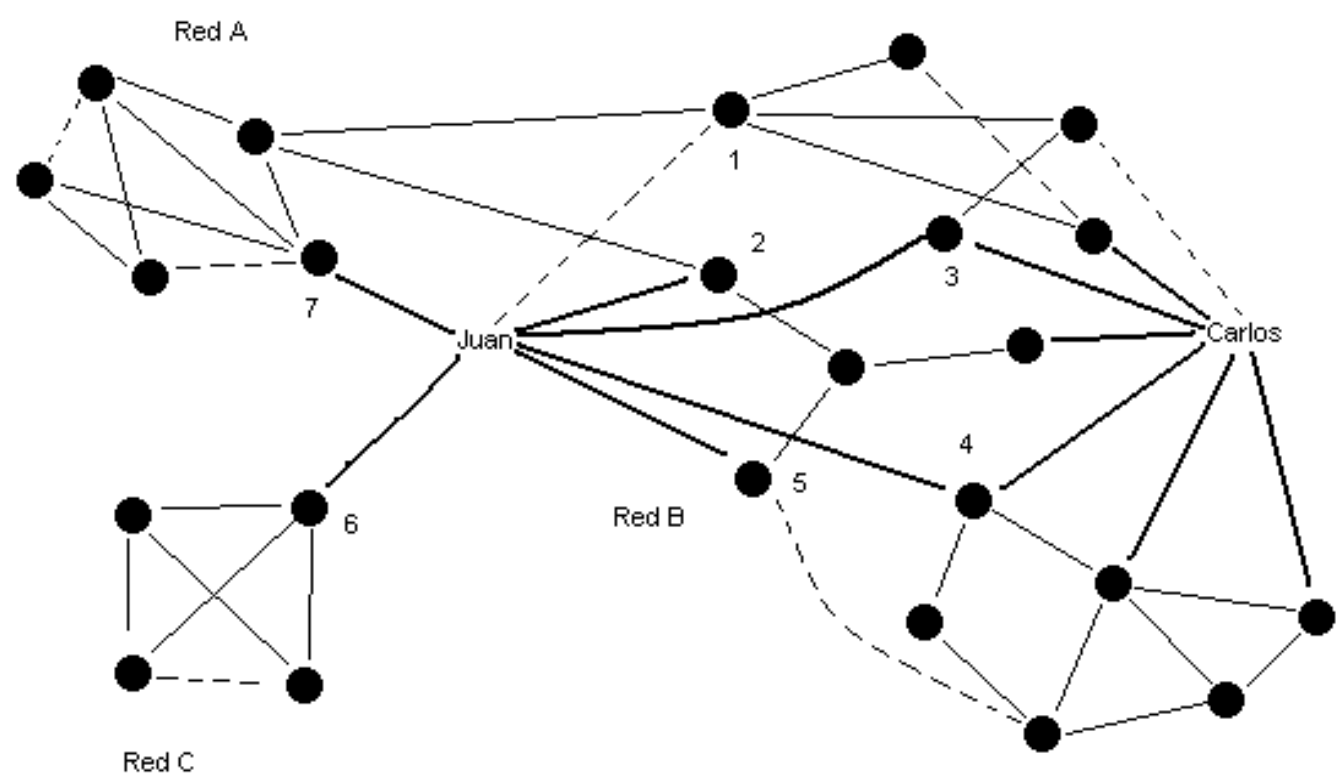

Figura 3. Red Social. Elaboración a partir de Burt (2005:14).

La información puede expandirse a través de la red alcanzando en diferentes momentos a todos los actores; pero la densidad más alta dentro de los clusters significa que la información circula más rápidamente dentro de los grupos que entre ellos (Burt, 2005:15). Esto puede dar lugar a diferencias en las creencias y

\footnotetext{
${ }^{1}$ Las personas suelen estar rodeadas de un núcleo de vínculos fuertes que les suministran recursos materiales y psíquicos cruciales para la supervivencia y la felicidad. Este núcleo fuerte está formado por un número pequeño de personas con las que se mantiene un contacto estrecho y frecuente, como la familia o los amigos. Junto a este círculo fuerte existe una gran cantidad de vínculos débiles con otras personas con las que el contacto es más superficial y menos frecuente, que pueden ser conocidos, vecinos o colegas.
} 
comportamientos entre los grupos, $\mathrm{y}$, por tanto, puede crear barreras para la información novedosa inconsistente con las opiniones y prácticas imperantes dentro del grupo. En este contexto, se definen huecos estructurales (structural holes) (Burt, 1992; 2001; 2004; 2005; Lin, Cook y Burt, 2001) como espacios vacíos entre los clusters de una red por los cuales la información no fluye, es decir, zonas vacías en la estructura de la información que fluye por la red. Las opiniones y los comportamientos varían más entre los grupos que dentro de ellos debido a los huecos estructurales en el flujo de información que atraviesa a la red. El valor potencial de los huecos estructurales consiste en que éstos separan fuentes de información no redundante, es decir, fuentes de información más aditivas que solapadas (Burt, 2005:16).

Consideremos dos actores sociales en la figura 3 tales como Carlos y Juan (ejemplo adaptado de Burt, 2005:16-19). Ambos individuos tienen el mismo número de relaciones: seis vínculos fuertes y uno débil; sin embargo, poseen diferentes entornos estructurales. El acceso de un individuo al capital social inserto en una red depende mucho más de su posición estructural en la red que del número de vínculos que posea. Carlos está conectado directamente a ciertos individuos del grupo $B, y$, a través de ellos, al resto de actores de dicho grupo. Juan está también conectado directamente a algunos individuos del grupo $\mathrm{B}, \mathrm{y}$, mediante ellos, a todos los miembros de tal grupo. Sin embargo, Juan está además conectado a los grupos $A$ y $C$. El vínculo de Juan con el nodo 7 es uno de los lazos entre los grupo $B$ y $\mathrm{A}$, pero el vínculo con el nodo 6 es el único lazo entre los clusters $\mathrm{B}$ y $\mathrm{C}$. También se puede definir puente estructural como aquel vínculo que si desaparece deja desconectados totalmente a dos grupos. Así pues, la relación de Juan con el nodo 6 es un puente estructural. Los puentes estructurales atraviesan los huecos estructurales permitiendo el flujo de información entre distintos clusters. Además, los puentes estructurales hacen posibles que las distancias que conectan a los individuos a través de los clusters sean pequeñas, $y$, por tanto, posibilitan que las redes sociales sean mundos pequeños.

En comparación con Carlos, Juan tiene tres ventajas derivadas de su posición en la estructura de la red social donde están insertos: acceso a una información más amplia, acceso temprano a esa información y control sobre la difusión de dicha información (Burt, 2005:17). En primer lugar, los vínculos-puente permiten a Juan acceder a una información más variada, es decir, menos redundante (más novedosa); en segundo lugar, la posición de Juan en un cruce de caminos en el flujo de información entre grupos le permite conocer nuevas ideas y comportamientos más rápidamente que otros. Así pues, Juan es lo que podría 
considerarse un líder de opinión, responsable de la difusión de la información en los clusters a los que pertenece. Además, Juan puede facilitar la comunicación entre clusters traduciendo opiniones y comportamientos entre los distintos lenguajes de diferentes grupos. Dicha labor de intermediación puede proporcionarle numerosas ventajas. Así pues, Juan es un intermediario (broker) o un emprendedor en el mundo de las redes sociales, y tiene más capital social derivado del brokerage que Carlos. Se puede concluir, por tanto, que individuos que viven en la intersección de mundos sociales suelen tener más información y mejores ideas que los que viven aislados en sus clusters.

Es importante subrayar de nuevo que la información que recibe un individuo no depende siempre del número de vínculos que posee; es decir, más vínculos no significan necesariamente más información. Para ilustrar este punto podemos considerar las siguientes dos redes de la figura 4.

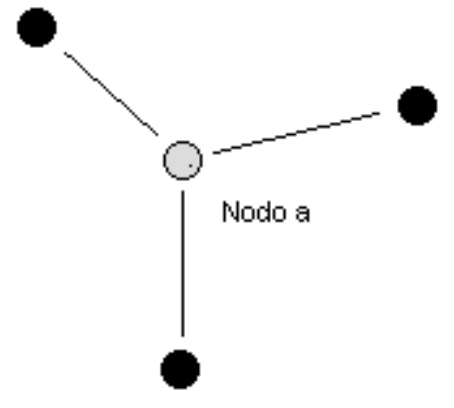

Red A

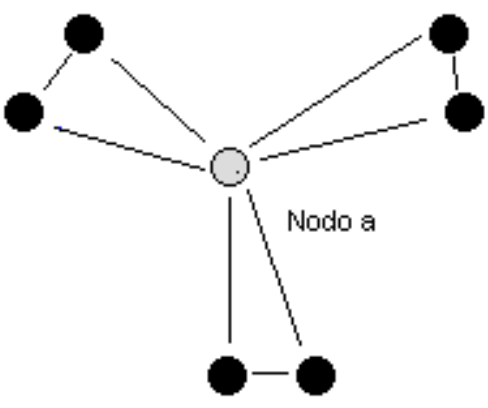

Red $\mathrm{B}$

Figura 4. Información redundante. Cohesión estructural.

En la red $\mathrm{A}$ el nodo a posee tres vínculos y en la red $\mathrm{B}$ el mismo nodo mantiene los tres vínculos anteriores y crea tres nuevos vínculos. Sin embargo, el nodo a no tiene más información en la red $A$ que en la $B$ porque las fuentes de información no son los nodos, sino los clusters. La red B proporciona la misma información que la red $A$ porque ambas redes poseen las mismas fuentes de información, pero el coste en tiempo y energía para mantener la red $B$ es el doble que para la red $A$ porque la red $\mathrm{B}$ tiene el doble de vínculos. Nuevos vínculos no implica necesariamente más información, pero si añaden siempre más costes. Supongamos, por ejemplo, que el individuo a trabaja en un empresa y necesita información de tres departamentos diferentes (tres clusters distintos) para realizar su trabajo. Contactar con más gente de cada departamento no le permite necesariamente desempeñar mejor su labor. Este aspecto estructural de la red B que proporciona información redundante se 
llama cohesión estructural: individuos fuertemente conectados entre sí (cluster) aportan información superflua.

Tampoco se puede afirmar que dos nodos que tengan el mismo número de vínculos dispongan de la misma información. Para reflexionar sobre esta idea podemos considerar las dos redes siguientes de la figura 5.

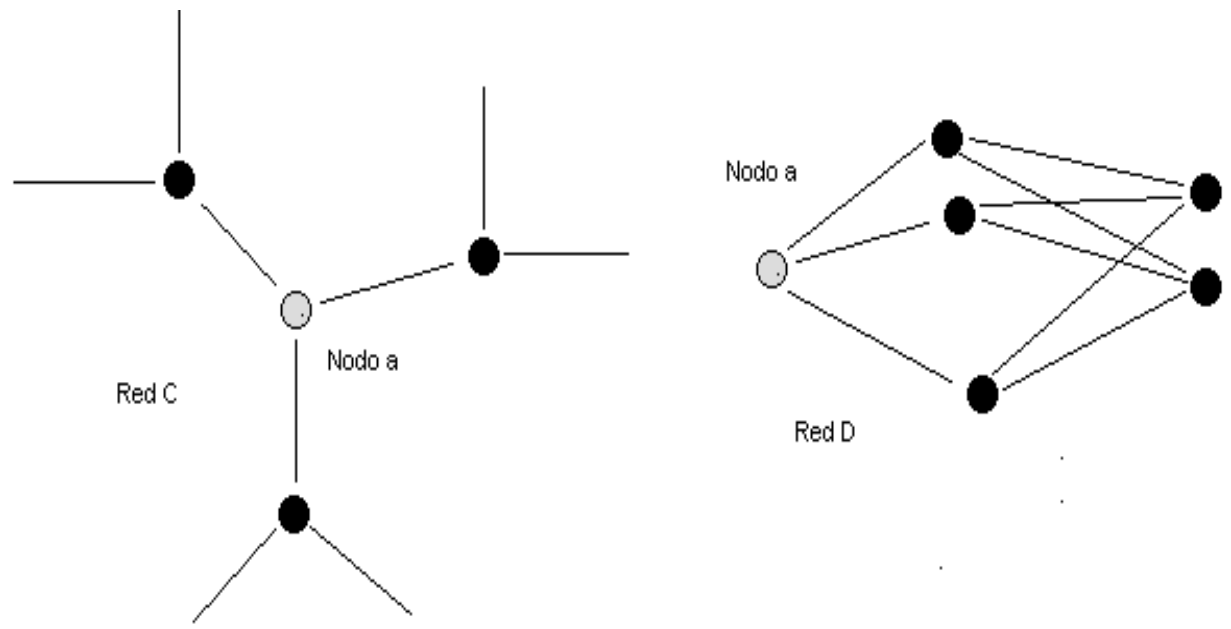

Figura 5. Redundancia estructural. Equivalencia estructural.

El nodo a tiene tres vínculos tanto en la red $C$ como en la $D$; sin embargo, la información que recibe en la red $\mathrm{C}$ es mayor que la suministrada por la red $\mathrm{D}$. Los nodos de color negro de la red $\mathrm{C}$ pertenecen a clusters distintos, pero los de la red D forman parte del mismo cluster. Por tanto, el nodo a dispone de más información en la red $C$ que en la $D$, o tiene menos información redundante. Los jefes de tres supermercados de ciudades distintas de la misma cadena de alimentación, por ejemplo, pueden que no se conozcan entre sí, pero habrán recibido posiblemente el mismo entrenamiento por parte de la dirección de la cadena. Por tanto, solucionarán los problemas de manera parecida. Este aspecto estructural de la red D que proporciona información redundante se llama equivalencia estructural: individuos desconectados entre sí pero unidos a la misma fuente reciben información similar (Burt, 2005:20).

Así pues, existen dos fuentes de información redundante, a saber, la cohesión estructural y la equivalencia estructural. Por tanto, redes con pocos vínculos, con vínculos demasiados interconectados (cohesión estructural) o con vínculos demasiado conectados indirectamente a través de un grupo central (equivalencia estructural) obtienen poca información o tienen información redundante, y, consecuentemente, poseen menos capital social derivado del brokerage. 


\section{b) Closure}

Supongamos ahora (figura 6) dos actores sociales tales como Carmen y Sonia, pertenecientes a dos segmentos de red distintos. Ellas han iniciado una relación (comercial, de trabajo o de cualquier otro tipo) que se puede calificar de débil por carecer de una historia común y no poseer un entorno social compartido. La confianza entre ellas cuando, por ejemplo, comparten información sensible será menor que si tuvieran una relación fuerte basada en el conocimiento mutuo y en la cooperación repetida durante tiempo, como se representa en la figura 7.

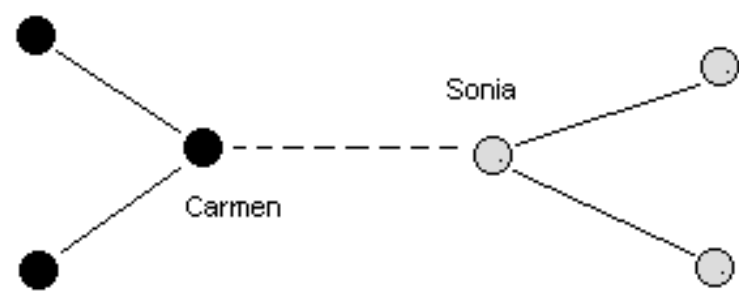

Red A

Figura 6: Relación débil: Conocidos

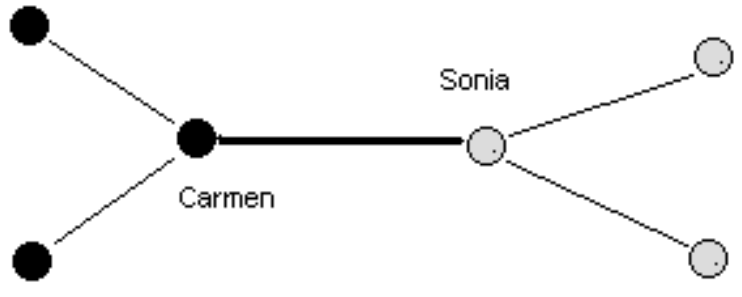

Red $\mathrm{B}$

Figura 7: Relación fuerte: Amigos

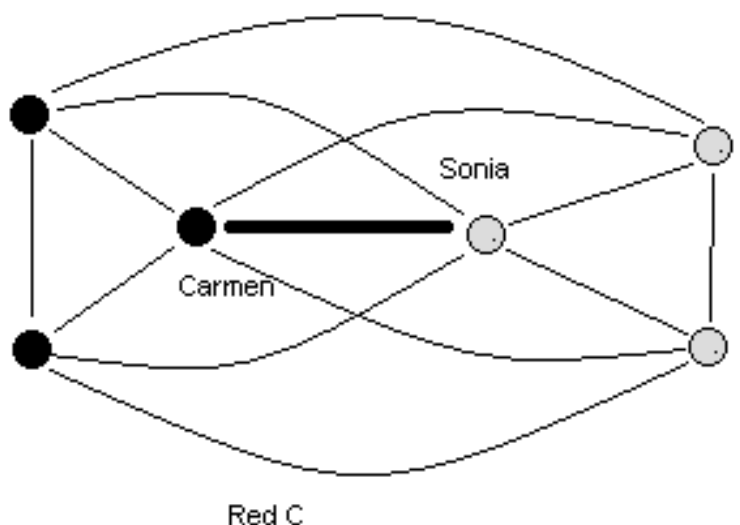

Figura 8: Red cerrada. Figuras elaboradas a partir de Burt (2005:96). 
Cuando la relación entre dos actores es de amistad, el riesgo de traición es menor que si fuese una relación de meros conocidos. Sin embargo, el riesgo de cometer alguna traición entre ellas es aún menor si existen conocidos y amigos comunes, como se observa en la figura 8. El sentimiento de culpa generado por engañar a un amigo de siempre puede ser enorme, incluso aunque la traición no sea descubierta (figura 7); pero el pesar puede ser aún mayor si dicha acción es descubierta por la víctima, y puede ser realmente insoportable si los amigos comunes se enteran de la "mala pasada" (figura 8).

El vínculo fuerte entre dos actores sociales se puede calificar de enlace (bond) cuando tiene lugar en medio de una red cerrada (cluster), es decir, dentro de una red constituida por nodos estrechamente y densamente conectados entre sí donde todo comportamiento público es conocido por todos los nodos (red C). El mal comportamiento de un actor en un grupo cerrado puede afectar gravemente a su reputación dentro del grupo. Así pues, en redes cerradas el riesgo de traición es menor $y$, consecuentemente, la confianza entre los miembros del grupo es mayor. Por tanto, la confianza derivada del closure en la red $C$ es probablemente mayor que en la red $B, y$, a su vez, la confianza en $B$ es seguramente mayor que en $A$.

El argumento podría ser resumido de la siguiente forma: dentro de una red cerrada y densa (constituida por agentes estrechamente y densamente conectados entre sí) todo comportamiento público es conocido por todos los agentes. Así pues, cuanto más cerrada y densa sea una red, más probable es que las malas conductas sean detectadas y, por tanto, castigadas. Asimismo, el mal comportamiento de un individuo puede influir enormemente en su reputación dentro del grupo cerrado y denso, $y$, por consiguiente, los individuos dentro de una red cerrada y densa poseen más tendencia a cooperar entre sí que en una abierta, ya que no quieren perder su reputación. El incentivo de la reputación para cooperar reduce la probabilidad de traición, $y$, por consiguiente, aumenta la posibilidad de confiar. Por idénticos motivos, en redes cerradas y densas la gente trabaja mejor, más tiempo y más eficazmente que en redes abiertas. Por tanto, no sorprende que los equipos unidos finalicen los trabajos más rápidamente, con más calidad y menos coste que la mera suma de individuos (Burt, 2005:162-163). En las organizaciones contemporáneas, el control de las cadenas de mando vertical y tradicional se ha sustituido por el closure que fuerza a los individuos a colaborar para mantener y aumentar la reputación dentro del grupo. 
Numerosas investigaciones empíricas ponen de manifiesto la existencia de capital social que emana del closure medido en forma de seguridad, identidad o felicidad. Coleman (1988; 1990:590-597), por ejemplo, señala que los jóvenes que viven en redes cerradas y densas de adultos tienen menos posibilidad de abandonar los estudios. Sus investigaciones indican, en primer lugar, que los jóvenes que viven en familias con dos padres y pocos hermanos tienen menos posibilidades de dejar el instituto que los que viven en familias monoparentales o con muchos niños. Padres que viven juntos son más efectivos en colaborar en la supervisión del estudiante que padres que viven separados. Segundo, jóvenes que han vivido en el mismo barrio toda la vida son menos propensos a dejar el colegio que chicos que han cambiado de vecindario. Padres, profesores y vecinos residentes en el mismo barrio de siempre probablemente se conozcan y puedan colaborar juntos en la supervisión de los jóvenes. Por último, los estudiantes de colegios privados son aun menos proclives a dejar el colegio porque este entorno favorece la comunicación y la colaboración entre padres y profesores para promover el adecuado desarrollo y el control de los jóvenes.

Otro importante estudio sobre el closure en redes sociales fue efectuado por Putnam (1993). En su investigación se analiza el éxito político de los gobiernos locales en ciertas regiones de Italia. Su hipótesis fue que redes sociales densas generan cultura cívica, y que la cultura cívica lleva al éxito político. Regiones con redes sociales densamente entretejidas en forma de ONGs, asociaciones de vecinos, cooperativas, clubes deportivos, partidos políticos y otras asociaciones tienen más cultura cívica y más capital social: "redes de compromiso cívico son una forma esencial de capital social: cuanto más densas sean las redes en una comunidad más probable es que sus ciudadanos puedan cooperar para mutuo beneficio" (Putnam, 1993:173). Además, este capital social es fundamental para el éxito político: "generar capital social no es una tarea fácil, pero es la clave para el funcionamiento de la democracia" (Putnam, 1993:115). El mecanismo de la reputación es la razón por la cual las redes densas incrementan la confianza entre las personas, promocionan las normas de reciprocidad, mejora el flujo de información sobre la honradez de los individuos, y encarnan éxitos cooperativos pasados que pueden servir como plantilla para futuras colaboraciones (Putnam, 1993:173-174).

En el grafo de la figura 9, las líneas continuas representan relaciones fuertes, las discontinuas simbolizan relaciones débiles y las líneas de puntos relaciones negativas. Consideremos un actor social tal como Martín (ejemplo adaptado de Burt, 2005:169-180). Dicho individuo tienen tres relaciones fuertes (con los nodos 
1,2 y 3 ), dos relaciones débiles (con los nodos 4 y 5 ), y dos relaciones negativas (con los nodos 6 y 7). Por todo lo dicho hasta ahora, Martín confiará más en sus vínculos fuertes que en los débiles, y confiará bastante menos en sus vínculos negativos: $(1,2,3)>(4,5)>(6,7)$

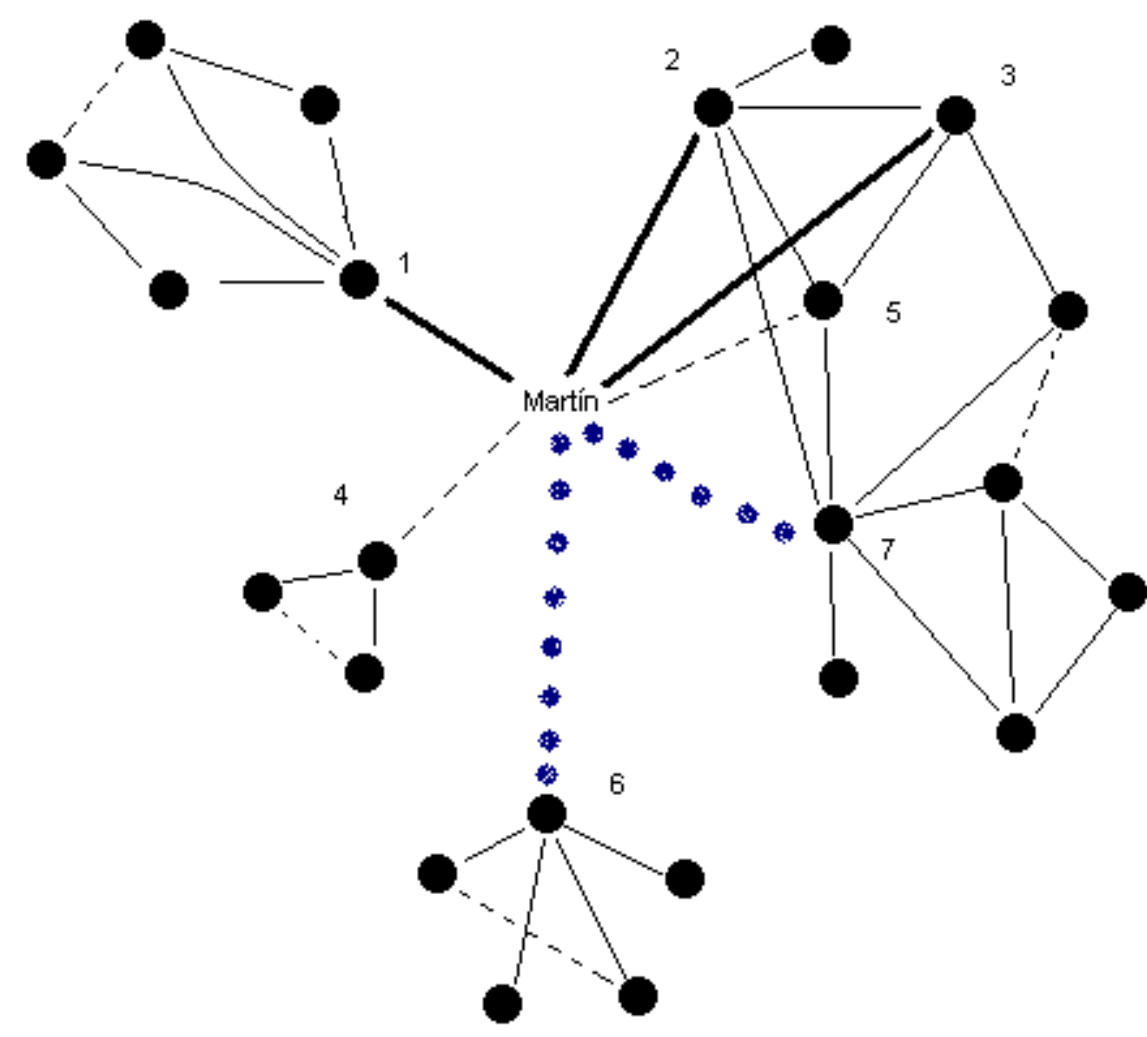

Figura 9. Red Social. Elaboración a partir de Burt (2005:169).

Pero entre sus vínculos fuertes confiará probablemente más en 2 y 3 que en 1 porque con 2 ó 3 tiene amigos comunes, y con el nodo 1 no hay ninguna amistad común. Entre los contactos débiles, Martín confiará probablemente más en 5 que en 4 porque con 5 tiene amigos comunes (los nodos 2 y 3), y con 4 no. Respecto a las relaciones negativas, Martín confiará seguramente más en 7 que en 6 porque su vínculo con 7 tiene algún amigo común, y con 6 no. Así pues el orden de confianza para el alter podría ser: $(2,3>1)>(5>4)>(7>6)$.

La información redundante en las redes con closure facilita las sanciones, y, por tanto, reduce el riesgo de traición entre miembros del grupo, aumentando entonces el nivel de confianza en el grupo; por el contrario, la información no redundante en las redes con brokerage genera nuevas ideas y comportamientos. Para el closure, la información valiosa es aquella que es redundante ya que la información repetida y consistente genera más confianza; para el brokerage, sin embargo, la información 
valiosa es la no redundante puesto que la información diversa y no repetida puede dar lugar a mejores ideas. Dicho con otras palabras, mientras que el valor del closure viene de la consistencia de la información dentro del grupo, los beneficios del brokerage proceden de la variación de creencias y prácticas entre los grupos (Burt, 2005:107). Los puentes estructurales son valiosos porque crean variación en la información y pueden generar nuevas ideas, mientras que los clusters son importantes porque eliminan la variación en las creencias y protegen a los individuos frente al riesgo de traición dentro del grupo. Así pues, hay una relación inversa entre brokerage y closure respecto a la información que fluye por las redes (el contenido de las redes sociales) (Burt, 2005:163): Juan tiene más capital social derivado del brokerage que Carlos (figura 4), pero Carlos posee más capital social emanado del closure que Juan (figura 9).

En conclusión, a lo largo de este epígrafe se ha mostrado cómo diferentes estructuras de redes (brokerage y closure) pueden crear distintos tipos de capital social como información y reciprocidad basada en la confianza. Además, se ha observado también cómo las posiciones estructurales de los individuos pueden dotar a dichos individuos de algunas ventajas en la realización de sus propias acciones: los actores mejor conectados tendrán mayores beneficios. La estructura de las redes aporta otro punto de vista para explicar y comprender las desigualdades sociales.

\section{Definición operativa de capital social}

Que el capital social se derive de las redes sociales no implica que las redes sociales sean capital social. Las redes sociales son fuentes potenciales de capital social, pero no son en sí mismas capital social (Herreros, 2002:10). Sin redes sociales no es posible el capital social, pero el capital social está más allá de las redes sociales. Confundir las fuentes del capital social con el capital social no sólo no permite un análisis en profundidad de dicho término, sino que impide responder de manera razonable a la cuestión sobre cómo elevar las reservas de capital social.

En este trabajo se consideran dos formas de capital social: el flujo de información que recorre la red a través de los puentes estructurales, y las obligaciones de reciprocidad que pueden surgir de las relaciones de confianza derivadas, a su vez, de los clusters. Este apartado tiene un doble objetivo: por un lado, proponer un mecanismo que relacione no solo la estructura de red (closure) sino también la acción individual con las obligaciones de reciprocidad, la confianza y el coeficiente de clustering; $y$, por otro lado, presentar un segundo mecanismo que ligue tanto la 
estructura de red (brokerage) como la acción de los actores con el flujo de información y la longitud de paso de la red.

\section{a) Obligaciones de reciprocidad}

Muchos teóricos del capital social (Burt, 2005; Putnam, 1993) asumen, sin más, que la confianza -en sus diversas definiciones- es capital social. Sin embargo, el hecho que la confianza entre individuos se derive del closure no significa necesariamente que la confianza sea capital social. La confianza no es capital social, pero puede generar recursos de capital social si logra crear obligaciones de reciprocidad, es decir, obtener favores a cambio de favores (Herreros, 2002:1018). Dicho lo anterior, cabe preguntarse cómo y a través de qué mecanismos la estructura de las redes sociales puede generar obligaciones de reciprocidad, y, por tanto, capital social. Responder esta cuestión nos lleva, en primer lugar, a analizar con más profundidad cómo la estructura de las redes y la acción individual pueden producir confianza; $y$, en segundo lugar, a investigar cómo la confianza puede generar obligaciones de reciprocidad.

Se tratará ahora de responder a la primera cuestión: cómo la estructura de las redes sociales y la acción individual son capaces de generar confianza. Supongamos que un individuo se enfrenta a la decisión de confiar o no en otro individuo sin tener la seguridad de que esa persona sea digna de confianza. Dicha decisión es una decisión bajo riesgo porque no conocemos las probabilidades objetivas de lo digno de confianza que es dicha persona. No obstante, aunque desconozcamos las probabilidades objetivas, podemos tener expectativas subjetivas acerca de la confianza que nos inspira dicha persona.

Consideremos que el individuo a se enfrenta a la decisión de confiar o no en el individuo $b$ (Alesina y La Ferrara, 2002). Supongamos que $p$ es la probabilidad subjetiva para el individuo a de que el individuo $b$ sea digno de confianza, y 1 - $p$ la probabilidad subjetiva de que no sea digno de confianza. Supongamos que $G$ es la ganancia potencial para el individuo $a$ en el caso de que el individuo $b$ decida honrar la confianza depositada en él, y $C$ el coste potencial para el individuo a en el caso de que el individuo $b$ traicione dicha confianza. Suponiendo que el individuo $a$ sea neutral ante el riesgo, es decir, que no tenga ni atracción ni fobia por el riesgo, dicho individuo $a$ decidirá confiar en el individuo $b$ si 


$$
\begin{aligned}
& \text { pG }-(1-p) C>0, \\
& \text { es decir, } \\
& \text { pG }>(1-p) C, \\
& y, \text { por tanto, } \\
& p /(1-p)>C / G
\end{aligned}
$$

Así pues, el individuo $a$, indiferente ante el riesgo, decidirá confiar en el individuo $b$ cuando se cumpla la anterior desigualdad (Coleman, 1990:99).

Si observamos la inecuación de Coleman acerca de la decisión de confiar o no en otra persona, podemos percatarnos de que dicha decisión depende de tres parámetros: las expectativas subjetivas acerca de lo digno de confianza que sea dicha persona $(p)$, de los beneficios $(G)$ y de los costes potenciales $(C)$ para la persona que se enfrenta con la decisión de confiar. Por consiguiente, aunque la probabilidad subjetiva de lo digno de confianza sea muy baja, esto es, aunque confiemos poco en la otra persona, es posible a pesar de todo arriesgarnos a confiar en él si las ganancias en el caso de que sea digno de confianza son muy altas en relación a las pérdidas en el caso de que nos traicione.

La estructura de una red puede influir en el cociente $C / G, y$, por tanto, afectar a la decisión de confiar. Para ilustrar esta influencia podemos considerar la relación entre $a$ y $d$ en la red de la figura 10 . Se puede observar que a tiene dos vínculos fuertes con $b$ y $c$ (los amigos), y un vínculo débil con $d$ (un conocido). Además, $a, b$ y $c$ forman un cluster (todos son amigos entre sí), y a y $d$ no tienen amigos comunes. Supongamos también que $a$ y $d$ son igualmente dignos de confianza, es decir, la probabilidad para a de que $d$ sea digno de confianza, $P a(d)$, es igual que la probabilidad para $d$ de que a sea digno de confianza, Pd (a). Sin embargo, las pérdidas que sufrirá $a$ al ser engañado por $d$ si deposita en él su confianza serán percibidas por a como potencialmente menores porque podrá basarse en $b$ y $c$ para compensarlas (Herreros, 2002:75). En cambio, las pérdidas de $d$ al ser engañado por a si deposita en él su confianza serán percibidas por $d$ como potencialmente mayores porque no posee ningún amigo extra. Esto es así porque la estructura de red que percibe a es distinta de la que experimenta $d$. Así pues, como en la decisión de a de confiar en $d$ el cociente $C / G$ es menor, a tenderá a confiar más en $d$ que $d$ en a porque en la decisión de $d$ de confiar en a el cociente $C$ / $G$ es mayor. Aquel que tiene más recursos experimentará unas pérdidas potenciales comparativamente menores que aquel que tiene menos recursos, $y$, por tanto, tenderá a confiar más (Herreros, 2002:75). Así pues, la posición que ocupan los 
individuos dentro de la estructura de una red es determinante a la hora de confiar. Independientemente de la fortaleza de este argumento, lo importante es señalar de nuevo que la estructura de una red puede influir en la decisión de confiar.

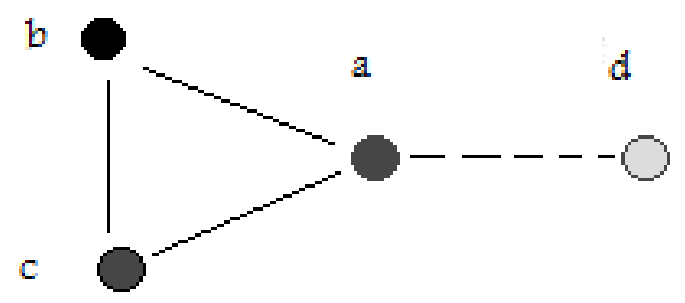

Figura 10. Relación de confianza.

Pero la estructura de una red también puede influir sobre $p \mathrm{y}$, por tanto, afectar a la decisión de confiar. Para ilustrar este hecho, consideremos otra vez la figura 10. La información para a acerca del resto de los individuos que pertenecen a su red social puede ayudarle a formar sus expectativas subjetivas sobre lo digno de confianza que son dichos individuos. Se puede observar que a tiene más información sobre $\mathrm{b}$ que sobre $d$ porque con $b$ tiene un amigo común $c$, y con $d$ no tiene a nadie en común. Así pues, la decisión de a de confiar o no en $b$ está mejor fundada que la decisión de a de confiar o no en $d$, puesto que a dispone de más información sobre $b$ que sobre $d$. Además, si en una red social alguien engaña a otra persona es de esperar que el engañado dé a conocer el comportamiento oportunista a otros miembros de la red, lo que probablemente destruirá la reputación del engañador. Por tanto, $a$ tenderá a engañar menos a $b$ que $d$ porque a se juega su reputación como individuo digno de confianza no sólo frente a $b$, sino también frente a $c$, mientras que frente a $d$, a sólo se juega su reputación con $d$. Por consiguiente, $a$ tenderá a confiar más en $b$ que en $d$ porque $b$ tiene que mantener una reputación frente a $c$ que $d$ no tiene que mantener. De este modo, podemos concluir que la posición que ocupen los individuos dentro de la estructura de red es crucial a la hora de confiar.

El coeficiente de clustering de una red es un parámetro estructural que mide el grado de extensión en el que los amigos de cada uno de los individuos de una red son entre sí mutuamente amigos. A medida que aumenta el coeficiente de clustering de una red, aumenta el número de vínculos entre los amigos de los individuos. Los individuos que pertenecen a redes con coeficientes de clustering altos (redes cerradas) tenderán a confiar más en otras personas de la red que los que pertenecen a redes con coeficientes de clustering bajos (redes abiertas) debido 
fundamentalmente a dos razones: primera, la probabilidad de ser digno de confianza $(p)$ en las redes cerradas es más alta que en las redes abiertas porque en la redes cerradas la presión para mantener la reputación de ser digno de confianza es mayor que en las redes abiertas; y segunda, el cociente $C / G$ en las redes cerradas es menor que en las redes abiertas porque en las redes cerradas los individuos poseen más recursos frente a las traiciones que en las redes abiertas.

Si la estructura de una red -caracterizada por diversos parámetros, entre los que se puede considerar el coeficiente de clustering- influye en la decisión de confiar, y la decisión de confiar -como se verá adelante- puede generar obligaciones de reciprocidad (uno de los aspectos del capital social), entonces se puede concluir nuevamente que la estructura de una red influye en la creación de capital social. Ahora bien, habiendo mostrado cómo la estructura afecta a la confianza, queda por mostrar cómo la confianza puede crear obligaciones de reciprocidad, esto es, obtener favores a cambio de favores.

La decisión de confiar de una persona $a$ en otra persona $b$ y pedirle un favor genera capital social cuando se crea la obligación en $a$ de devolver dicho favor a $b$ si ésta se lo pide, y cuando se genera la obligación en $b$ de honrar esa confianza que $a$ le ha depositado de no traicionarlo (Herreros, 2002:12-17). Que a devuelva el favor a $b$ puede ser una acción racional porque mantener la reputación de ser digno de confianza es fundamental para que en el futuro puedan hacerle más favores; y que $b$ honre la confianza depositada y no traicione también puede ser una acción racional porque preserva de igual manera la reputación. Por tanto, la decisión de confiar genera obligaciones de reciprocidad (un elemento del capital social) si está mediada por la reputación (Herreros, 2002:13).

En este sentido, las redes cerradas (caracterizadas por el closure) pueden crear más capital social (entendido como obligaciones de reciprocidad) que las redes abiertas porque los individuos que pertenecen a redes cerradas tienden a ser más confiados que los que pertenecen a redes abiertas. Asimismo, los actores que participan en las redes cerradas (altos valores del coeficiente de clustering) toman sus decisiones con más confianza que en las redes abiertas (bajos valores del coeficiente de clustering) porque en las redes cerradas se obtiene más fácilmente información acerca de la reputación de los individuos, y además porque los individuos pueden sobreponerse mejor a las traiciones que en las redes abiertas (Burt, 2005:93-166; Lin, 2001:66). 


\section{b) Información}

El flujo de información que recorre las redes sociales es otra forma de capital social (Burt, 2005; Coleman, 1990:310). Las redes sociales que en principio se mantienen con otros fines, poseen como subproducto la obtención de información (Herreros, 2002:18). La participación en una asociación puede suministrar información sobre temas alejados de los objetivos concretos de la asociación: al formar parte de una asociación de astronomía, por ejemplo, se puede obtener sin duda información sobre planetas y estrellas, y disfrutar con los compañeros de la observación del cielo nocturno; pero también el compañero de al lado -que es economista- puede proporcionar información interesante sobre las ofertas de trabajo del banco en el que trabaja. Esta información es capital social, un recurso que se obtiene como subproducto de la participación en una asociación.

La estructura de red también influye en el flujo de información que recorre la red, como se ha mostrado en páginas anteriores. En las redes cercanas (bajos valores de la longitud de paso), aquellas donde los individuos están conectados al resto de los nodos por pocos pasos, la información se transmite más rápidamente que en las redes lejanas (altos valores de la longitud de paso), donde los actores están enlazados al resto por muchos pasos.

En las figuras 11 y 12 se ilustran respectivamente una red cercana y otra lejana. Los individuos de la red cercana están conectados como máximo por dos pasos; en cambio, los individuos de la red lejana están enlazados por un máximo de cuatro pasos. En las redes cercanas la obtención de la información es más fácil que en las redes lejanas porque la información tarda menos en difundirse por la red, es decir, las redes de pocos pasos disponen más rápidamente de capital social (entendido como información) que las redes de muchos pasos. Además, la calidad de la información que circula por las redes cercanas es probablemente mayor que la que circula por las redes lejanas porque, en el proceso de transmisión, la información va perdiendo calidad conforme aumenta el número de pasos (principio entrópico). Asimismo, la posición que ocupe un individuo en la red es clave no sólo para la rapidez con que se consigue la información, sino también para la calidad de información que se obtiene. El individuo de color gris de la red cercana obtiene más fácilmente información y de mejor calidad de su periferia que el individuo de color gris de la red lejana. 


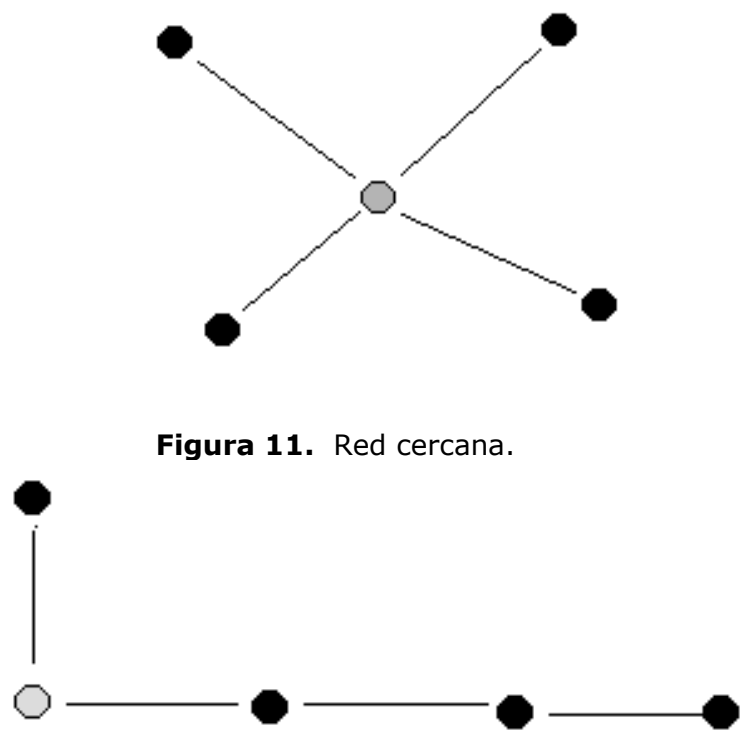

Figura 12. Red lejana.

La longitud de paso de red es un parámetro estructural que mide el grado de cercanía entre los individuos de una red. A medida que disminuye la longitud media de paso, más cercanos se encuentran los individuos entre sí, y, por tanto, la información es de más calidad y fluye más rápidamente. Por consiguiente, los individuos que pertenecen a redes cercanas (Iongitud de paso baja) obtendrán más fácilmente información y de más calidad que los individuos que pertenecen a redes lejanas (longitud de paso alta). Así pues, podemos concluir que la estructura de una red -caracterizada en este caso por la longitud de paso- influye en la rapidez con que se difunde el flujo de información y en la calidad con que se obtiene, es decir, en la génesis de capital social.

\section{Conclusión}

Podemos terminar este artículo resumiendo brevemente los argumentos expuestos. Me he centrado en dos aspectos del capital social, a saber, las obligaciones de reciprocidad y la información. Por una parte, ciertas estructuras de las redes sociales (closure) pueden generar confianza entre los individuos que pertenecen a dichas redes (Burt, 2005), y dicha confianza puede producir obligaciones de reciprocidad a través de un mecanismo: el mantenimiento de la reputación (Herreros, 2002). Por otro lado, otras estructuras de las redes sociales (brokerage) pueden facilitar el flujo de información que atraviesan tales redes (Burt, 2005). Ambas formas de capital social son recursos accesibles para todos aquellos que participan en las estructuras de las redes sociales (Lin, 2001). 
También se ha mostrado cómo las propiedades estructurales de las redes sociales afectan a la creación de capital social. Por una parte, el coeficiente de clustering influye en la confianza entre los individuos de la red que puede generar obligaciones de reciprocidad y, por otro lado, la longitud de paso afecta al flujo de información que pasa por la red. Por tanto, ambos parámetros estructurales influyen en la génesis de capital social: a medida que se incremente el coeficiente de clustering o que disminuya la longitud de paso de una red, aumentan las posibilidades de generar más capital social.

En este artículo se ha analizado, pues, cómo ciertas propiedades estructurales de las redes sociales influyen en la génesis de capital social, pero también sería posible investigar cómo otros aspectos estructurales pueden afectar a la creación de capital social. Así pues, en el futuro se podría indagar cómo otras propiedades de gran trascendencia sociológica tales como el grado de desigualdad en la distribución de vínculos, la centralidad, K-grupos, por ejemplo, afectan a la transmisión de información y a la creación de obligaciones de reciprocidad, es decir, a los recursos de capital social.

\section{Bibliografía}

Alesina, A. and E. La Ferrara (2002). "Who Trusts Others?", Journal of Public Economics 85:207-234.

Andréu, J. comp. (2005). Desde la esquina de Europa. Análisis comparado del capital social en Andalucía, España y Europa. Sevilla: Centro de Estudios Andaluces.

Barabási, A. L. (2002). Linked. The New Science of Networks. Cambridge, MA: Perseus.

Bourdieu, P. (1986). "The forms of Capital." Pp. 240-268 in Handbook of theory and research for the sociology of education, edited by J. G. Richardson. New York: Greenwood.

Burt, S. R. (1992). Structure Holes: The Social Structure of Competition. Cambridge, MA: Harvard University Press.

Burt, S. R. (2001). "Structural Holes versus Network Closure as Social Capital." Pp. 31-56 in Social Capital: Theory and Research, edited by N. Lin, K. S. Cook, and R. S. Burt. New York: Aldine de Gruyter.

Burt, S. R. (2004). "Structural holes and good ideas", American Journal of Sociology 110: 349-399.

Burt, S. R. (2005). Brokerage and Closure. Oxford: Oxford University Press. 
Coleman, J. S. (1988). "Social Capital in the Create of Human Capital", American Journal of Sociology 94:95-120.

Coleman, J. S. (1990). Foundations of Social Theory. Cambridge: Harvard University Press.

Coleman, J. S., H. Menzel and E. Katz (1957). "The diffusion of an innovation among physicians", Sociometry 20:253-270.

Erickson, B. H. (2001). "Good networks and Good Jobs: The value Social Capital to Employers and Employees." Pp. 127-158 in Social Capital: Theory and Research, edited by N. Lin, K. S. Cook and R. S. Burt. New York: Aldine de Gruyter.

Gambetta, D. ed. (2000). Trust: Making and Breaking Cooperative Relations. Department of Sociology, University of Oxford: Electronic Edition.

González-Bailón, S. (2006). "The Role of Dynamic Network in Social Capital: A Simulation Experiment", Papers: Revista de Sociología 80:171-194.

Granovetter, M. (1973). "The strength of weak ties", American Journal of Sociology 76: 1360-1380.

Granovetter, M. [1974] (1995). Getting a Job. Chicago: University of Chicago Press. Hanneman, R. A. y M. Riddle (2005): Introduction to social network methods, Riverside, CA: University of California, Riverside.

Herreros, F. (2002). ¿Por qué Confiar? El Problema de la Creación de Capital Social. Madrid: CEACS.

Kawachi, I., S. V. Subramanian and D. Kim. eds. (2007). Social Capital and Health. New York: Springer.

Levi, M. (1996). "Social and Unsocial Capital: A Review Essay of Robert Putnam's Making Democracy Work", Politics and Society 24(1):45-55.

Lin, N. (1982). "Social Resources and Instrumental Action." Pp. 132-145 in Social Structure and Network Analysis, edited by P. V. Marsden and N. Lin. Beverly Hills, CA: Sage.

Lin, N. (2001). Social Capital. A Theory of Social Structure and Action. Cambridge: Cambridge University Press.

Lin, N., K. S. Cook and R. S. Burt. eds. (2001). Social Capital: Theory and Research. New York: Aldine de Gruyter.

Layard, R. (2005). Happiness. Lessons from a New Science. London: Penguin Books. 
Marx, K. [1849] (1933). Wage-labour and Capital. New York: International Publishers.

Molina, José Luis (2001): El análisis de las redes sociales. Una introducción, Barcelona: Bellaterra.

Narayan, D. ed. (2002). Empowerment and Poverty Reduction. Washington, DC: The World Bank.

Newton, K. (1997). "Social Capital and Democracy", American Behavioural Scientist 40(5):575-586.

Ostrom, E. and J. Walker. eds. (2005). Trust and Reciprocity: interdisciplinary lessons from experimental research. New York: Russell Sage Foundation.

Paxton, P. (1999). "Is Social Capital Declining in the United States? A Multiple Indicator Assessment", American Journal of sociology 105:88-127.

Portes, A. (1998). "Social Capital: Its Origins and applications in Modern Sociology", Annual Review of Sociology 22:1-24.

Putnam, R. D. (1993). "The Prosperous Community: Social Capital and Public Life", The America Prospect 13 (spring):35-42.

Putnam, R. D. (1995). "Bowling Alone: America's Declining Social Capital", Journal of Democracy 6:65-78.

Putnam, R. D. (2000). Bowling Alone. The collapse and revival of American community. New York: Simon and Schuster.

Putnam, R. D. y K. A. Goss. (2003). "Introducción", en El declive del capital social compilado por R. D. Putnam. Barcelona, Círculo de lectores.

Requena, Félix (1989): "El concepto de red social", Revista Española de Investigaciones Sociológicas, 48: 137-152.

Smith, A. (1937). The Wealth of Nations. New York: Modern Library.

Velásquez, A., y L. Rey (2007). "El valor agregado de las redes sociales: propuesta metodológica para el análisis del capital social", Redes. Revista hispánica para el Análisis de Redes Sociales, 13 (5).

Wasserman, S. y K. Faust (1994): Social Network Analysis, Cambridge: Cambridge University Press.

Watts, D. J. (2003). Six Degrees: the Science of a connected Age. New York: Norton. 
Woolcock, M. (1998). "Social Capital and Economic Development", Theory and Society 27:151-208.

Woolcock, M., and D. Narayan (2000). "Social Capital: Implications for Development Theory, Research and Policy", The World Bank Observer 15 de August: 225- 249. 